\title{
The early history of protostellar disks, outflows and binary stars
}

\author{
Dennis F. Duffin \\ Department of Physics and Astronomy, McMaster University, Hamilton ON L8S 4M1, Canada \\ duffindf@mcmaster.ca \\ and \\ Ralph E. Pudritz \\ Department of Physics and Astronomy, McMaster University, Hamilton ON L8S 4M1, Canada \\ Origins Institute, McMaster University, Hamilton ON L8S 4M1, Canada \\ pudritz@physics.mcmaster.ca
}

\begin{abstract}
In star formation, magnetic fields act as a cosmic angular momentum extractor that increases mass accretion rates onto protostars and in the process, creates spectacular outflows. However, recently it has been argued that this magnetic brake is so strong that early protostellar disks - the cradles of planet formation - cannot form. Our three-dimensional numerical simulations of the early stages of collapse $\left(\lesssim 10^{5} \mathrm{yr}\right)$ of overdense star-forming clouds form early outflows and have magnetically regulated and rotationally dominated disks (inside 10 AU) with high accretion rates, despite the slip of the field through the mostly neutral gas. We find that in three dimensions, magnetic fields suppress gravitationally driven instabilities which would otherwise prevent young, well ordered disks from forming. Our simulations have surprising consequences for the early formation of disks, their density and temperature structure, the mechanism and structure of early outflows, the flash heating of dust grains through ambipolar diffusion, and the origin of planets and binary stars.
\end{abstract}

Subject headings: binaries: general — circumstellar matter — methods: numerical — stars: formation — stars: general — stars: low-mass, brown dwarfs

\section{Introduction}

Over the past decade numerical simulations have enabled the exploration of the central physical questions regarding the nature of star formation - from the collapse of an initial dense gaseous molecular cloud core to the formation of a star and its associated protostellar disk and outflow, and the emergence of a star's planetary system. The formation of disks and jets during star 
formation is central to many of these issues, but very little is known about the earliest phases of their evolution. How is the initial excessive angular momentum associated with the star's natal core removed - through magnetic braking (Basu \& Mouschovias 1994) and then outflows (Baneriee \& Pudritz 2006; Pudritz et al. 2007), or by spiral density waves in disks (e.g. Larson 2009)? Do multiple stars form through gravitational fragmentation of cores or massive disks (Hosking \& Whitworth 2004; Price \& Bate 2007; Hennebelle \& Tevssier 2008)? What is the significance of outflows and jets that are launched before most of the mass has collapsed into the disk (Lynden-Bell 2003; Baneriee \& Pudritz 2006) in a wide variety of young stellar systems from brown dwarfs (Whelan et al. 2005; Machida et al. 2009) to massive stars (Arce et al. 2007; Banerjee \& Pudritz 2007)?

Extraction of angular momentum by magnetic fields that thread the collapsing gas may be too efficient, according to recent two-dimensional (Mellon \& Li 2008) and three-dimensional axisymmetric (Hennebelle \& Fromang 2008) simulations, preventing the formation of rotationally dominated disks, even when the effects of imperfect coupling of the field with the gas are included (Mellon \& Li 2009). These results seemingly contradict the observations which clearly show that disks are present around most if not all young stars, even in environments in which the magnetic field is expected to be strong (Hartmann \& Calvet 1995). Hennebelle \& Fromang (2008) performed three-dimensional ideal MHD simulations of collapsing cores using a barotropic equation of state, concluding that no rotationally dominant structure is formed from 10 to 100 AU for highly magnetized cores. Mellon \& Li (2008) performed two-dimensional ideal MHD simulations on collapsing singular isothermal toroids using a barotropic equation of state and an inner boundary at $6.7 \mathrm{AU}$ (effectively a sink particle), finding that even moderately magnetized disks could not form. Such two-dimensional models impose a high degree of mathematical symmetry and therefore miss the formation of bars and spiral waves in disks.

We improve on previous results through this three-dimensional adaptive mesh refinement (AMR) investigation which includes a full treatment of the cooling (Banerjee et al. 2006) and the finite coupling of the magnetic field to the pre-stellar gas (ambipolar diffusion, Duffin \& Pudritz 2008). We find that ordered disk-like structures can emerge on scales $\lesssim 10$ AU at early times $\left(\lesssim 10^{5}\right.$ yr) in magnetized systems. We have omitted a sink particle in this study as they are expected to affect the solution to within a few sink radii. Without sinks simulations are indeed limited to early disks, although they offer a full solution to the region within 10 AU where the heated core forms.

\section{Methods}

We model an isolated star forming core with a rotating Bonnor-Ebert (BE) sphere (Bonnor 1956; Ebert 1955) commonly observed in nature (Alves et al. 2001; Lada et al. 2003; Teixeira et al. 2005), using the FLASH AMR code. The refinement criteria resolves the Jeans' length $\left(\lambda_{\mathrm{J}}=\right.$

$\sqrt{\pi c_{s}^{2} / G \rho}$ by at least eight cells, limited by the ambipolar diffusion time step. The ambipolar 
diffusion time step is

$$
\tau_{\mathrm{AD}}=0.5 \frac{\Delta x^{2}}{\eta_{\mathrm{AD}}}=0.5 \frac{\mu_{0} \gamma_{\mathrm{AD}} \rho_{i} \rho_{n} \Delta x^{2}}{1.4 B^{2}},
$$

where $\Delta x$ is the cell length, $\eta_{\mathrm{AD}}$ is the diffusivity, $\gamma_{\mathrm{AD}}=3.28 \times 10^{1} 3 \mathrm{~g}^{-1} \mathrm{~cm}^{3} \mathrm{~s}^{-1}$ is the ion-neutral collisional coupling, $B$ is the magnetic field strength, and $\rho_{i}=2.32 \times 10^{-25}\left(\rho_{n} / 10^{-18}\right)^{0.5} \mathrm{~g} \mathrm{~cm}^{-3}$ (e.g. Duffin \& Pudritz 2008).

We construct an initial BE sphere with a radius of $R=6.9 r_{0}=6.9 c_{s_{\text {core }}} / \sqrt{4 \pi G \rho_{c}}$ (where the critical radius is $R_{c}=6.49 r_{0}$ ) and add a $10 \%$ over-density on top with a $10 \%, m=2$ perturbation to break axisymmetry. Such perturbations model the buffeting that observed star-forming cores undergo from supersonic turbulent gas motions that prevail in the surrounding low density molecular gas (our perturbation accounts for a $0.04 \%$ change in gravitational binding energy, so this is a rather quiescent core). Our BE spheres have a mass of $1.18 M_{\odot}$ and initial central densities of $\rho_{c}=6.07 \times 10^{-18} \mathrm{~g} \mathrm{~cm}^{-3}$. The magnetic field is added to the core in the $z$ direction - in agreement with recent observations (Kirk et al. 2009) - such that the ratio of the thermal to the magnetic energy in the gas, known as the plasma beta $\left(\beta=2 c_{s}^{2} / v_{\mathrm{A}}^{2}=46.01\right)$ is constant, where $c_{s}=0.27 \mathrm{~km} \mathrm{~s}^{-1}$ is the core's sound speed and the Alfvn speed is $v_{\mathrm{A}}=B / \sqrt{4 \pi \rho} \simeq 0.74 c_{s} / \Gamma$ (the latter relation for critical BE spheres). The mass-to-flux ratio $\Gamma$ is a measure of the ratio of the gravitational to the magnetic energy in gravitating objects. If $\Gamma<1$, the magnetic field is strong enough to prevent gravitational collapse and the core is called sub-critical, otherwise it is called supercritical. We set the mass-to-flux $\Gamma=2 \pi G^{1 / 2} \Sigma / B=3.5$, where $\Sigma$ is the column density, stemming from observations of magnetic fields in early cores which indicate $\Gamma \approx 1-4$ (Crutcher 1999) and confirmed through simulations of cloud-scale turbulence (Tilley \& Pudritz 2007). The rotation values of our models were taken from previous simulations of early star-forming clusters (Tilley \& Pudritz 2007). We take the extremes and average values of rotation and interpret these as low, moderate and high rotating cores (Table 1). The moderate rotation model set has a ratio of rotational to gravitational binding energy of $\beta_{\text {rot }}=0.046$, in line with observations (Lada et al. 2003).

For each model set, we run a non-magnetic (hydrodynamic), a perfectly coupled (ideal magnetohydrodynamic) and more realistic, partially coupled (ambipolar diffusion) case for a total of nine simulations (summarized in Table 1).

\section{Do disks form?}

By the time the maximum surface densities in the moderate rotation model set reach a value of $\Sigma_{c}=4.2 \times 10^{3} \mathrm{~g} \mathrm{~cm}^{-2}, 0.1 M_{\odot}$ is contained within $156 \mathrm{AU}$ (about $10 \%$ of the core's mass is contained within $0.003 \%$ of its initial volume). The mass of the protostar (taken to be all material inside $1 \mathrm{AU}$ or $10^{-3} M_{\odot}$ ) is only $6 \%$ of the total mass in the disk (taken to be everything inside 10 $\mathrm{AU}$ or $\left.10^{-2} M_{\odot}\right)$. At this point, the non-magnetic, perfectly coupled and partially coupled clouds are $0.160,0.194$, and 0.175 million years old respectively (corresponding to 5.9, 7.2 and 6.5 free-fall times, where $t_{\mathrm{ff}}=0.027 \mathrm{Myr}$ is estimated using $\rho_{c}$ ). An extended dense region has taken shape, 
the gas has been heated, and in the cases where magnetism is present, disk winds have started.

We present in Figure 1, snapshots of the structure of the region at the center of the collapse, taken at this time for our three different cases. Despite having identical initial conditions, the non-magnetized, moderate rotation case has formed two bars, driving off-axis rotational modes which themselves create an off-axis bar. The wobbling in the collapse occurs through accretion along shocks and density perturbations in the gas. This stands in stark contrast to the obvious disk-like structures and outflows present in both ambipolar diffusion (Figure 1(b) and ideal MHD cases (Figures 1(c) and (d) 1 . Strong oscillations of the disk radius were noted in the simulations of Price \& Bate (2007) which were also dominated by massive disks in their early phase. They also found that this behavior was suppressed by magnetic fields.

Most importantly, the magnetic disks, by preventing strong bars from forming, are rotationally dominated whereas the uncoupled disk is not! This is illustrated in Figure 2(a), where we see that the ambipolar diffusion case has a larger rotationally dominated disk $\left(v_{\phi}>v_{\text {infall }}\right)$ than the perfectly coupled case. We note also, by plotting $v_{\phi} / v_{\text {infall }}$ of the most evolved perfectly coupled state of the collapse, that the rotational support is growing with time while hydrodynamic rotational support becomes increasingly unstable. In Figure 2(a) we also plot surface averaged plots of the ratio of the rotational velocity to the Keplerian velocity - the rotational velocity of a gas in a stable orbit around an enclosed mass. We find peak ratios of about $0.1-0.5$ in all cases of the moderate rotation model (in fact some pre-binned values exceed 1 in the ambipolar case).

In the limit of low rotation, hydrodynamic spiral waves and bars in the purely hydrodynamic case are confined to a region of about $1 \mathrm{AU}$ in radius. This has little effect on the braking of the cloud (Figure 2(d) and rotationally dominated disks are allowed to form outside of 1 AU (Figure 2(b). The hydrodynamic case is much more spherical in nature while the magnetized cases remain fairly flat, despite efficient magnetic braking. In the limit of low rotation, Figure 2(b) shows that the rotation in the hydrodynamic case is nearly Keplerian and the magnetic case is much more sub-Keplerian, whereas in the case of moderate rotation shown in Figure 2(a) the opposite is true. Thus in the extreme low rotation limit where spiral modes and bars are strongly suppressed, early hydrodynamic disks do form.

Due to the bar, the distribution of specific angular momentum $j(R)$ (where $R$ is the cylindrical radius; Figure 2 (d) of the hydrodynamic case is comparable to the cases where magnetic braking is present. In the low rotation model set, the bar is small and $j(R)$ does not compare as well to the magnetized cases. The hydrodynamic and perfectly coupled cases of the moderate rotation model set have similar maximal accretion rates of $\dot{M}_{\text {accr }} \approx 4 \times 10^{-4} \mathrm{M}_{\odot} \mathrm{yr}^{-1}$, while the ambipolar case reaches maximal accretion rates of only $\dot{M}_{\text {accr }} \approx 1 \times 10^{-4} \mathrm{M}_{\odot} \mathrm{yr}^{-1}$ due to weaker magnetic braking and a stable disk. In the low rotation model set, mass accretion rates are $\dot{M}_{\text {accr }} \approx 2 \times 10^{-4} \mathrm{M}_{\odot} \mathrm{yr}^{-1}$ for the magnetized cases and $\dot{M}_{\text {accr }} \approx 3 \times 10^{-4} \mathrm{M}_{\odot} \mathrm{yr}^{-1}$ for the hydrodynamic case.

\footnotetext{
${ }^{1}$ We provide movies of Figures 1(a)-(c) online illustrating the density and magnetic field line structure on all scales in the simulation.
} 
In the high rotation model set however, no cases form rotationally dominated disks as all three cases have formed some sort of bar on large scales (Figure 3). Clearly, it is through the suppression of instabilities in the collapse that magnetic fields promote early disk formation. Disk formation in systems without magnetic fields typically must wait until the mass of the central star dominates that of the surroundings sufficiently to suppress these instabilities.

\section{Early outflows and magnetic fields}

Figures 1(b)-(d) are snapshots of the structure of outflows that are launched at these early times. A major result, shown by comparing Figures 1(b) and (c), is that outflows occur even when ambipolar diffusion is active. Outflows in a non-ideal MHD collapse have been demonstrated in twodimensional ambipolar diffusion without drift heating (Mellon \& Li 2009) and in three-dimensional using an ohmic diffusion approximation (Machida et al. 2007). Our analytical understanding of the early outflow mechanism is the magnetic tower (Lynden-Bell 2003). In this picture, the toroidal field in the disk is generated through the rotating flow and confined there by the accretion ram pressure. As the field winds up, it reaches a critical strength in which the toroidal pressure is strong enough to overcome the ram pressure, moving wrapped field lines away from the disk and taking perfectly coupled gas with them - starting with the initial release in Figure 1(c) and the more developed magnetic bubble shown in Figure 1(d). This acts to torque down the disk, removing angular momentum and increasing accretion rates. In the ambipolar diffusion picture, these field lines can seep through the ram pressure as they are not so tightly coupled to the accreting gas.

We have also discovered the existence of a centralized, high-speed component to the ambipolar diffusion case's outflow ( $v>0.4 \mathrm{~km} \mathrm{~s}^{-1}$ in an region roughly 1 AU in size). This component resembles a jet that is commonly observed in the evolved perfectly coupled collapse 3 . We note that the base of this component to the outflow lies on a cushion of heat generated by the friction between ions and neutrals as toroidal field lines seep through the accreting gas. This spike in drift heating is typical in ambipolar diffusion mediated C-shocks, regions where ion-neutral friction is strong (Wardle 1991). This local heating effect produces strong gradients in temperature with $T>110$ $\mathrm{K}$ in regions roughly $5 \mathrm{AU}$ in size, both above and below the midplane. Within these regions are features roughly $1 \mathrm{AU}$ in size with $T>1000 \mathrm{~K}$ (not unheard of in $\mathrm{C}$-shocks). By comparison, the perfectly coupled outflow has a sharp temperature shock at about $50 \mathrm{~K}$ leading its outflow. In contrast, the low rotation model set shows very little drift heating and a similar weak outflow (Figure 2(c). While the vertical field threading the disk $B_{z}$ is identical for all low and moderate rotation, magnetized cases (Figure 2(f), the toroidal field $B_{\phi}$ in the low rotation, magnetized cases is nearly 2 orders of magnitude smaller than in the moderate rotation, magnetized cases.

In Figure 2(c) we plot the ratio of mass loss rates $\dot{M}_{\text {wind }}$ to mass accretion rates $\dot{M}_{\text {accr }}$ for

\footnotetext{
${ }^{2}$ A movie of the development of Figure 1(d) from Figure 1(c) is provided online.

${ }^{3}$ The velocity structure of the perfectly coupled outflow is seen in our online movie of the evolution of Figure 1(d)
} 
the moderate and low rotation model sets. For comparison we also plot this ratio for the evolved outflow of the perfectly coupled case at later times (labeled 'evolved ideal'). The evolved outflow is very efficient in the moderate rotation, ideal MHD case - the central outflow component exceeds speeds of $5 \mathrm{~km} \mathrm{~s}^{-1}$ and observed outflow rates of $\dot{M}_{\text {wind }} / \dot{M}_{\text {accr }} \sim 0.1$ (Hartmann \& Calvet 1995). Unfortunately, we cannot fully develop the ambipolar outflow much beyond $10^{5} \mathrm{yr}$ due to time step constraints. However, from this early footprint, we are led to believe that the outflow mechanism is working. In the low rotation, ideal MHD case, the outflow rates are significantly diminished by nearly 2 orders of magnitude in comparison to mass accretion rates, less than what is typically observed. This may have consequences on feedback effects in star forming clusters and the assembly of massive stars (Wang et al. 2009); protostellar energy injection is dependent on the rotational energy of the star forming core.

\section{Fragmentation and binaries}

In supercritical cores, three-dimensional ideal MHD calculations have shown that strong perturbations (on the order of 50\%) are needed in order for cores with $\Gamma \sim 2$ to fragment and form binaries, even with high rotation (Price \& Bate 2007; Hennebelle \& Teyssier 2008). We find that none of the low and moderate rotation cases, magnetized or not, fragment. We therefore ran a high rotation model set with four times the angular rotation than our earlier model set (roughly 20 times the rotational energy, an extreme rotation (Tilley \& Pudritz 2007)), similar to the simulations of Price \& Bate (2007) with a 10\% perturbation amplitude. In Figure 3 we plot three-dimensional density contours for all three cases of the high rotation model at the largest common central column density $\left(\Sigma_{c}=68 \mathrm{~g} \mathrm{~cm}^{-2}\right)$. The hydrodynamic case quickly fragments into a binary with a wide separation of roughly 1000 AU. The perfectly magnetized case forms a bar and does not fragment, stabilized through its magnetic pressure as well as through the effects of magnetic braking. Meanwhile, the ambipolar diffusion case produces an intermediate result. Early on a ring is formed which fragments into a bar-like object with a small companion at about 1000 AU.

For sufficiently high rotation ambipolar diffusion allows binaries to form without the need of strong (e.g. $\gtrsim 10 \%$ in amplitude) perturbations. For moderate rotation rates however, fragmentation can take place only if large perturbation amplitudes $(\approx 50 \%)$ are used in the initial conditions. Such a perturbation would amount to a $1 \%$ change in our BE sphere's gravitational binding energy. This relatively low level of 'turbulent' energy has been measured in B68 (Lada et al. 2003). This would suggest that a low level of turbulent energy (e.g. a few percent of the gravitational energy) is required in order to reproduce the overwhelming tendency for quiescent cores to form binaries in face of strong magnetic support. 


\section{Conclusions}

In the early stages of a purely hydrodynamic collapse of a moderately rotating core, material joins the protostar by accreting through a chaotic series of bars and spiral waves. Our results show that in magnetized collapses however, the magnetic field suppresses these wave modes, and a small, regular disk appears at the earliest times. The weakening of magnetic control by ambipolar diffusion is insufficient to guarantee the formation of binary stars in typical cores with moderate rotation. Our results suggest that modest turbulent amplitudes ( $>10 \%)$ appear to be required. Regarding planet formation, the central density structure of early disks (Figure 2(e) falls off as $\Sigma \propto r^{-(1.7-2.5)}$ much more quickly with disk radius than do protoplanetary disk models at later times, wherein $\Sigma \propto r^{-1.5}$ or $r^{-1}$. As the collapse winds up the field early outflows appear - even for partially coupled disks - and feed angular momentum and mechanical energy back into the star forming neighborhood. The density and magnetic structure of ideal and partially coupled disks are quite similar - the main difference is in the strength of the wound up magnetic field (at least an order of magnitude weaker in the ambipolar diffusions case).

Finally, we note that evidence for early localized intense drift heating near the disk at the accretion shock, which heats materials up to $1,000 \mathrm{~K}$ and beyond in a localized region, may be preserved in the observed composition of comets and meteorites. The rapid heating, and subsequent cooling of those crystalline Mg-rich silicate materials that passed through the accretion shock and through the region of high ambipolar heating is reminiscent of heating events that must have occurred for some of these materials seen in cometary grains (Wooden et al. 2007). These events have been attributed to shock heating by spiral waves out to $10 \mathrm{AU}$ in disks (Desch et al. 2005). As seen in Figure 1(b), only a portion of the accreted disk material will pass through this localized heated region. Subsequent radial turbulent mixing of this flash heated material with the bulk of material that passed through a more gentle heating environment could in principle contribute to the wide mixture of thermal histories preserved in cometary grain materials.

\section{Acknowledgements}

We thank Robi Banerjee, Eve C. Ostriker, and James Wadsley for fruitful discussions. D.D. was supported by McMaster University and R.E.P. by the Natural Sciences and Engineering Research Council of Canada. We are pleased to acknowledge the SHARCNET HPC Consortium for the use of its facilities at McMaster University. The software used in this work was in part developed by the DOE-supported ASC/Alliances Center for Astrophysical Thermonuclear Flashes at the University of Chicago. This research was supported in part by the National Science Foundation under grant PHY05-51164.

\section{REFERENCES}

Alves, J. F., Lada, C. J., \& Lada, E. A. 2001, Nat, 409, 159 
Arce, H. G., Shepherd, D., Gueth, F., Lee, C.-F., Bachiller, R., Rosen, A., \& Beuther, H. 2007, in Protostars and Planets V, ed. B. Reipurth, D. Jewitt, \& K. Keil, 245-260

Banerjee, R., \& Pudritz, R. E. 2006, ApJ, 641, 949

—. 2007, ApJ, 660, 479

Banerjee, R., Pudritz, R. E., \& Anderson, D. W. 2006, MNRAS, 373, 1091

Basu, S., \& Mouschovias, T. C. 1994, ApJ, 432, 720

Bonnor, W. B. 1956, MNRAS, 116, 351

Crutcher, R. M. 1999, ApJ, 520, 706

Desch, S. J., Ciesla, F. J., Hood, L. L., \& Nakamoto, T. 2005, in Astronomical Society of the Pacific Conference Series, Vol. 341, Chondrites and the Protoplanetary Disk, ed. A. N. Krot, E. R. D. Scott, \& B. Reipurth, 849-+

Duffin, D. F., \& Pudritz, R. E. 2008, MNRAS, 391, 1659

Ebert, R. 1955, Zeitschrift fur Astrophysik, 37, 217

Hartmann, L., \& Calvet, N. 1995, AJ, 109, 1846

Hennebelle, P., \& Fromang, S. 2008, A\&A, 477, 9

Hennebelle, P., \& Teyssier, R. 2008, A\&A, 477, 25

Hosking, J. G., \& Whitworth, A. P. 2004, MNRAS, 347, 1001

Kirk, J. M., Crutcher, R. M., \& Ward-Thompson, D. 2009, ApJ, 701, 1044

Lada, C. J., Bergin, E. A., Alves, J. F., \& Huard, T. L. 2003, ApJ, 586, 286

Larson, R. B. 2009, ArXiv e-prints

Lynden-Bell, D. 2003, MNRAS, 341, 1360

Machida, M. N., Inutsuka, S.-i., \& Matsumoto, T. 2007, ApJ, 670, 1198

-. 2009, ApJ, 699, L157

Mellon, R. R., \& Li, Z.-Y. 2008, ApJ, 681, 1356

—. 2009, ApJ, 698, 922

Price, D. J., \& Bate, M. R. 2007, MNRAS, 377, 77

Pudritz, R. E., Ouyed, R., Fendt, C., \& Brandenburg, A. 2007, in Protostars and Planets V, ed. B. Reipurth, D. Jewitt, \& K. Keil, 277-294 
Teixeira, P. S., Lada, C. J., \& Alves, J. F. 2005, ApJ, 629, 276

Tilley, D. A., \& Pudritz, R. E. 2007, MNRAS, 382, 73

Wang, P., Li, Z.-Y., Abel, T., \& Nakamura, F. 2009, ArXiv e-prints

Wardle, M. 1991, MNRAS, 251, 119

Whelan, E. T., Ray, T. P., Bacciotti, F., Natta, A., Testi, L., \& Randich, S. 2005, Nature, 435, 652

Wooden, D., Desch, S., Harker, D., Gail, H.-P., \& Keller, L. 2007, in Protostars and Planets V, ed. B. Reipurth, D. Jewitt, \& K. Keil, 815-833

This preprint was prepared with the AAS LATEX macros v5.2.

\begin{tabular}{l|rrr}
\hline \hline Model Set & Low Rotation & Moderate Rotation & High Rotation \\
\hline$\Omega t_{\mathrm{ff}}=\Omega \sqrt{3 \pi / 32 G \rho_{0}}$ & 0.1 & 0.3 & 1.2 \\
Angular rotation $\left(\Omega, \mathrm{s}^{-1}\right)$ & $1.18 \times 10^{-13}$ & $3.52 \times 10^{-13}$ & $1.41 \times 10^{-12}$ \\
Rotational beta $\left(\beta_{\text {rot }}\right)$ & 0.0052 & 0.046 & 0.74
\end{tabular}

Table 1: Model parameters. Each model set has a mass of $M_{\text {core }}=1.18 M_{\odot}$, core temperature of $T_{\text {core }}=20 \mathrm{~K}\left(c_{s_{\text {core }}}=0.267 \mathrm{~km} \mathrm{~s}^{-1}\right)$, external temperature of $T_{\text {ext }}=200 \mathrm{~K}\left(c_{s_{\text {ext }}}=0.845 \mathrm{~km} \mathrm{~s}^{-1}\right)$, $\Gamma=3.5$, and $\beta=46.01$ and is run in three cases: hydrodynamic, ideal MHD, and ambipolar diffusion. 

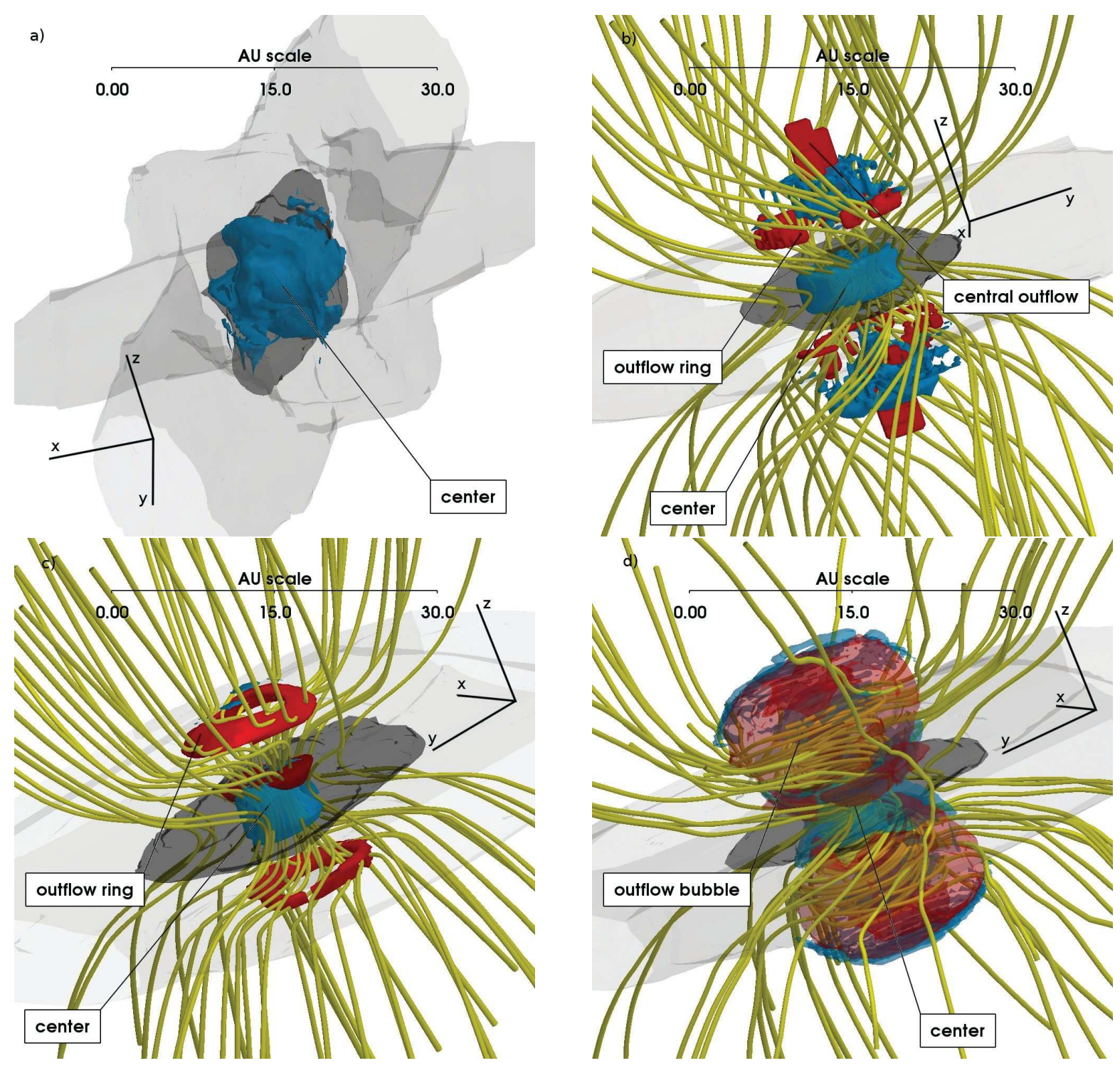

Fig. 1. - Inner disk of the moderate rotation model set at central surface densities of $\Sigma_{0}=$ $4.2 \times 10^{3} \mathrm{~g} \mathrm{~cm}^{-2}$ for (a) hydrodynamic, (b) ambipolar diffusion, (c) perfectly coupled cases, and (d) the evolved state of the perfectly coupled case at $\Sigma_{c}=8.72 \times 10^{7} \mathrm{~g} \mathrm{~cm}^{-2}$. Black and gray surfaces are $\rho=10^{-12} \mathrm{~g} \mathrm{~cm}^{-3}\left(n=2.58 \times 10^{11} \mathrm{~cm}^{-3}\right)$ and $\rho=10^{-13} \mathrm{~g} \mathrm{~cm}^{-3}\left(n=2.58 \times 10^{10} \mathrm{~cm}^{-3}\right)$, respectively. Temperature contours at $T=85 \mathrm{~K}$ are shown in blue. Outflow velocities contours, shown in red, are $0.3 \mathrm{~km} \mathrm{~s}^{-1}$ in (c) and (d) and $0.01-0.4 \mathrm{~km} \mathrm{~s}^{-1}$ in b. Magnetic field lines are shown as yellow tubes. (Animations of this figure are available in the online journal.) 

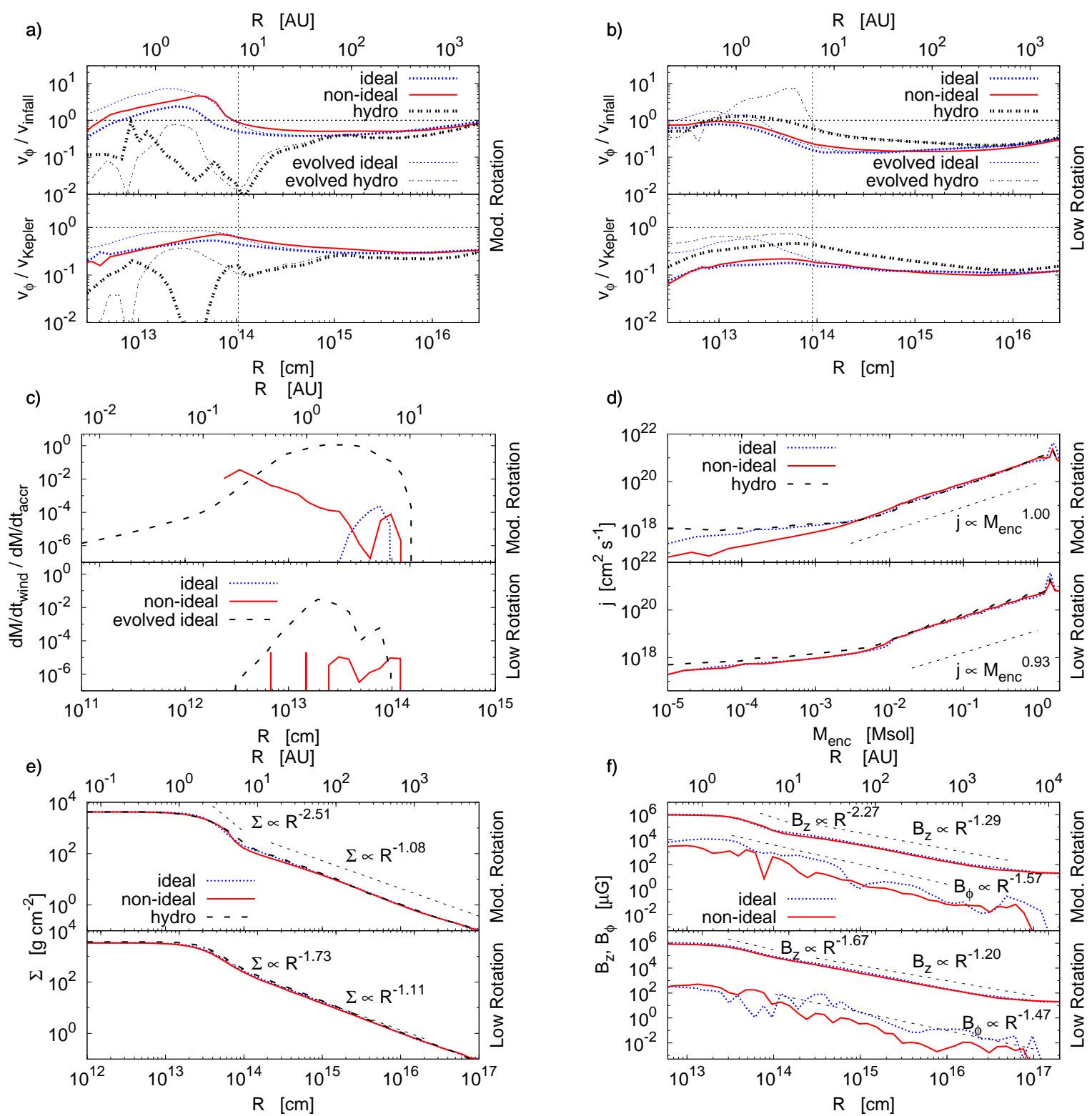

Fig. 2.- Azimuthally averaged plots (density weighted, see Banerjee \& Pudritz 2006) vs. disk radius for ideal $\mathrm{MHD}$, non-ideal $\mathrm{MHD}$, and hydro cases. In all figures the model sets are shown at a common central surface density of $\Sigma_{c}=4.2 \times 10^{3} \mathrm{~g} \mathrm{~cm}^{-2}$ (moderate or mod. rotation) and $\Sigma_{c}=3.4 \times 10^{3} \mathrm{~g} \mathrm{~cm}^{-2}$ (low rotation), when viewed face on. Panels (a) and (b) show the toroidal velocity in units of infall velocity and Keplerian velocity for the moderate and low rotation model sets, respectively ("evolved" refers to hydro and ideal MHD end-states). The dotted horizontal lines represent a velocity ratio of 1 . The dotted vertical lines represent a transition radius of 7 and 6 AU, respectively. Panel (c) shows the outflow efficiency of the magnetized cases as compared to the more evolved ideal MHD case. Panel (d) shows specific angular momentum plotted against enclosed mass. The surface density distributions are shown in panel (e), while the magnetic field distributions are shown in panel (f). 
a)

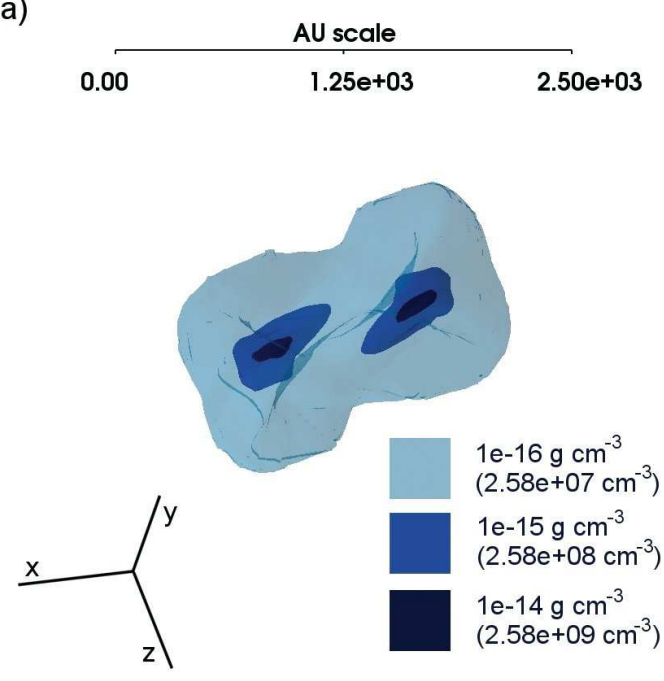

b)
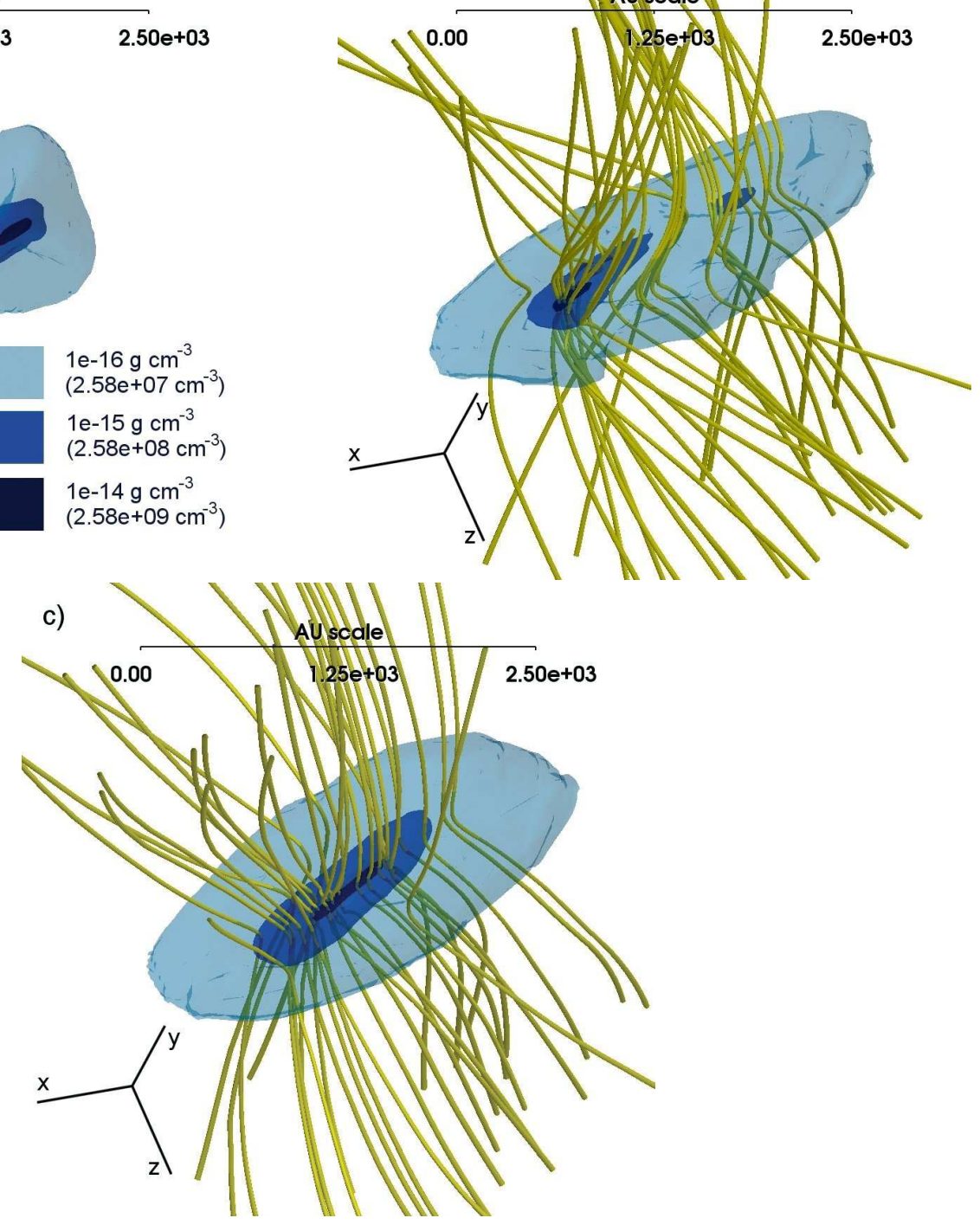

Fig. 3. - Large scale fragmentation of the high rotation model set for (a) hydrodynamic, (b) ambipolar diffusion, and (c) ideal MHD cases at similar scales and orientations. Density contours are given in the legend, and magnetic field lines are drawn as yellow tubes. 